\title{
BMJ Open Qualitative and quantitative assessment of posterior segment optical coherence tomography images using standard photos: the Liwan Eye Study
}

\author{
Sean K Wang, ${ }^{1}$ Xinxing Guo, ${ }^{2}$ Ou Xiao, ${ }^{2}$ Yanxian Chen, ${ }^{2}$ Ran Liu, ${ }^{2}$ \\ Wenyong Huang, ${ }^{2}$ Mingguang $\mathrm{He}^{2,3}$
}

To cite: Wang SK, Guo X, Xiao 0, et al. Qualitative and quantitative assessment of posterior segment optical coherence tomography images using standard photos: the Liwan Eye Study. BMJ Open 2017;7:e017923. doi:10.1136/ bmjopen-2017-017923

- Prepublication history and additional material for this paper are available online. To view these files, please visit the journal online(http://dx.doi. org/10.1136/bmjopen-2017017923).

Received 27 May 2017 Revised 16 0ctober 2017 Accepted 27 October 2017

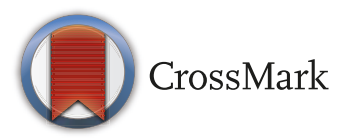

${ }^{1}$ Department of Medicine, Harvard Medical School, Boston, Massachusetts, USA

${ }^{2}$ State Key Laboratory of Ophthalmology, Zhongshan Ophthalmic Center, Sun Yat-sen University, Guangzhou, China

${ }^{3}$ Centre for Eye Research Australia; Ophthalmology, Department of Surgery, University of Melbourne, Melbourne, Australia

Correspondence to Dr Mingguang He; mingguang_he@yahoo.com

\section{ABSTRACT}

Background/aims To develop a standardised grading scheme, using standard photos, for spectral-domain ocular coherence tomography (SD-OCT) images of the posterior eye and evaluate the interobserver agreement among trained ophthalmologists in identifying pathological changes.

Methods Subjects were recruited from Liwan District, Guangzhou, with SD-OCT data collection from June 2013 to November 2013 as part of 10-year follow-up visits from the Liwan Eye Study. All subjects underwent SD-OCT imaging of the macula with scanning lines analysed by two ophthalmologists to assess for the presence of 12 different posterior segment lesions. Per cent agreement for each lesion between the graders and quantitative measures of dome-shaped macula (DSM) height and choroidal thickness were calculated.

Results A total of 679 SD-OCT images from 679 subjects were independently evaluated by the two graders. Each of the 12 lesions was successfully graded as present or absent in over $96 \%$ of images. For all lesions, per cent agreement between observers was over $90 \%$, ranging from $90.7 \%$ for epiretinal membranes and retinal pigment epithelium thickenings to $99.7 \%$ for full thickness macular holes and retinal detachments. Quantitative measurements of DSM height and choroidal thickness at three locations of the eye all exhibited intraclass correlation scores between the two graders of greater than 0.9 .

Conclusion Our study demonstrates high concordance between graders in characterising posterior segment lesions using SD-OCT images, validating the continued use of this imaging modality in the diagnosis of posterior eye disease.

\section{INTRODUCTION}

First introduced in 1991, optical coherence tomography (OCT) is a non-invasive imaging modality based on interferometry, able to generate real-time cross-sectional maps of the retina. ${ }^{1}$ Analogous to B-scan ultrasound, OCT divides light from a broadband light source into sample and reference beams and measures the interference pattern following backscatter from the back of the eye, ${ }^{2}$
Strengths and limitations of this study

- The study proposes and validates a set of standardised criteria for grading posterior segment spectral-domain ocular coherence tomography images in accordance with International Nomenclature for Optical Coherence Tomography consensus anatomic landmarks.

- The study assessed for 12 different pathological changes of the vitreoretinal interface, neurosensory retina and retinal pigment epithelium/Bruch's complex and presents standard photos for each.

- The study reports interobserver variability between trained ophthalmologists in grading posterior segment lesions, analogous to how these images are interpreted in most medical settings.

- Ocular coherence tomography images were obtained from a population-based study, resulting in low prevalence for several of the types of lesions.

resulting in a reflectivity versus depth profile of the retina useful in diagnostic decisions. Recently, widespread adoption of spectraldomain OCT (SD-OCT) technology has effectively replaced previous slower time-domain OCT devices, allowing for image acquisition rates of up to 29000 axial scans per second with a $6 \mu \mathrm{m}$ resolution or 14500 scans per second with $3.5 \mu \mathrm{m}$ resolution. ${ }^{3}$ With the advent of swept-source OCT using longer bandwidth light sources and higher resolution photodetectors, these numbers will only continue to improve.

Natural variation in reflectivity of normal retinal tissue underlies the basis of OCT to resolve the multiple layers comprising the vitreoretinal interface, neurosensory retina, retinal pigment epithelium (RPE)/ Bruch's complex and inner choroid. While nerve fibres and the RPE demonstrate high reflectivity, plexiform and nuclear layers show medium reflectivity, and photoreceptors display low reflectivity. ${ }^{45}$ In contrast to 
many subfields of medicine in which a tissue in question can readily be biopsied, ophthalmology and particularly the study of the retina are disproportionately dependent on imaging. Consequently, in clinical practice, OCT has become a routine and invaluable test to identify an extensive array of lesions ranging from epiretinal membranes (ERMs) and macular holes to pathological changes in the RPE.

OCT reading guidelines to date have been proposed for a number of conditions including glaucoma, diabetic maculopathy and age-related macular degeneration, ${ }^{6-8}$ but a comprehensive OCT classification scheme for the posterior segment changes has not yet been developed. In 2015, Heng et a $\theta^{\dagger}$ demonstrated a high percentage concordance between two retinal specialists in evaluating features of diabetic macular oedema. Similarly, in 2016, Kim $e t a l^{7}$ quantified the intergrader agreement among glaucoma specialists of using OCT images in the structural diagnosis of glaucoma. However, for many ocular lesions detectable on OCT, the reliability in grading among the specialists who diagnose them has not yet been characterised.

The purpose of this study was to develop a standardised grading scheme, using standard photos, for SD-OCT images of the posterior eye and to evaluate the interobserver agreement among trained ophthalmologists in identifying pathological changes. Although the applications of OCT in managing ocular disease have been widely celebrated, only recently has consensus terminology for normal posterior segment SD-OCT imaging been proposed, ${ }^{10}$ and little has been reported on the interobserver variability in assessing posterior segment disease by SD-OCT. Here, we define and investigate the grading of 12 different posterior eye lesions by SD-OCT to determine areas of agreement among the images collected in the Liwan Eye Study, a population-based study.

\section{METHODS \\ Subjects}

All subjects were enrolled from Liwan District, Guangzhou, with OCT data collection from June 2013 to November 2013 as part of 10-year follow-up visits from the Liwan Eye Study. The methodology of Liwan Eye Study has been described in detail elsewhere. ${ }^{11}$ In short, all subjects aged 50 years and older residing in the selected study clusters in Liwan District of Guangzhou for more than 6 months were enrolled in 2003. Written informed

\begin{tabular}{|c|c|}
\hline Lesion & Definition \\
\hline \multicolumn{2}{|l|}{ Vitreoretinal interface } \\
\hline Vitreomacular adhesion & $\begin{array}{l}\text { Elevation of the vitreous body above the macular area with remaining attachment of the } \\
\text { vitreous to the fovea }{ }^{21} \text { (figure } 1 \mathrm{~A} \text { ). }\end{array}$ \\
\hline Vitreomacular traction & $\begin{array}{l}\text { Vitreofoveal adhesion, distortion of the foveal surface and elevation of the foveal floor }{ }^{21} \\
\text { (figure 1B). }\end{array}$ \\
\hline Epiretinal membrane & Highly reflective layer on or above the inner surface of the retina ${ }^{22} 23$ (figure $1 C$ ). \\
\hline \multicolumn{2}{|l|}{ Neurosensory retina (layers $1-13$ ) } \\
\hline Full thickness macular hole & $\begin{array}{l}\text { Defect of the neurosensory retina affecting all layers from the internal limiting membrane } \\
\text { to the ellipsoid zone }{ }^{24} \text { (figure } 2 \mathrm{~A} \text { ). }\end{array}$ \\
\hline Lamellar macular hole & $\begin{array}{l}\text { Irregular fovea with partial loss of the inner layers of retina but with intact photoreceptor } \\
\text { layer and RPE/Bruch's complex } \\
\text { 25 (figure } 2 \mathrm{~B} \text { ). }\end{array}$ \\
\hline Macular retinoschisis & $\begin{array}{l}\text { Single or multiple separations within neurosensory retina, either between different layers } \\
\text { or within one neurosensory layer }{ }^{26} \text { (figure } 2 \mathrm{C} \text { ). }\end{array}$ \\
\hline Retinal detachment & Separation of neurosensory retina from the $\mathrm{RPE}^{27} 28$ (figure 2D). \\
\hline Macular oedema & $\begin{array}{l}\text { (1) Increased macular thickness throughout all layers of the retina, (2) focal thickening } \\
\text { with or without intraretinal cyst formation or (3) thickness exceeding }>300 \mu \mathrm{m} \text { on any } \\
\text { sector of the macular tomographic map }{ }^{29} 30 \text { (figure } 2 \mathrm{E}, \mathrm{F} \text { ). }\end{array}$ \\
\hline Intraretinal hyper-reflective lesion & $\begin{array}{l}\text { Hyper-reflective area in the neurosensory retina with or without shadowing effect on the } \\
\text { underlying outer retina and choroid }{ }^{31} \text { (figure } 2 \mathrm{G} \text { ). }\end{array}$ \\
\hline \multicolumn{2}{|l|}{ RPE/Bruch's complex (layer 14) } \\
\hline RPE thickening & $\begin{array}{l}\text { Regions of increased thickness in the RPE that tend to be localised and irregular }{ }^{32} \\
\text { (figure } 3 \mathrm{~A} \text { ). }\end{array}$ \\
\hline Pigment epithelium detachment & $\begin{array}{l}\text { Separation of the RPE from Bruch's membrane, with accumulation of sub-RPE fluid, } \\
\text { blood, fibrovascular tissue or lipoprotein-derived debris (figure 3B). }\end{array}$ \\
\hline Dome-shaped macula & $\begin{array}{l}\text { Inward bulge of the RPE layer resulting in a 'dome-like' protrusion }>50 \mu \mathrm{m} \text { from peak to } \\
\text { base above a presumed line tangent to the outer surface of the RPE }{ }^{33} 34 \text { (figure } 3 C \text { ). }\end{array}$ \\
\hline
\end{tabular}

RPE, retinal pigment epithelium; SD-OCT, spectral-domain optical coherence tomography. 

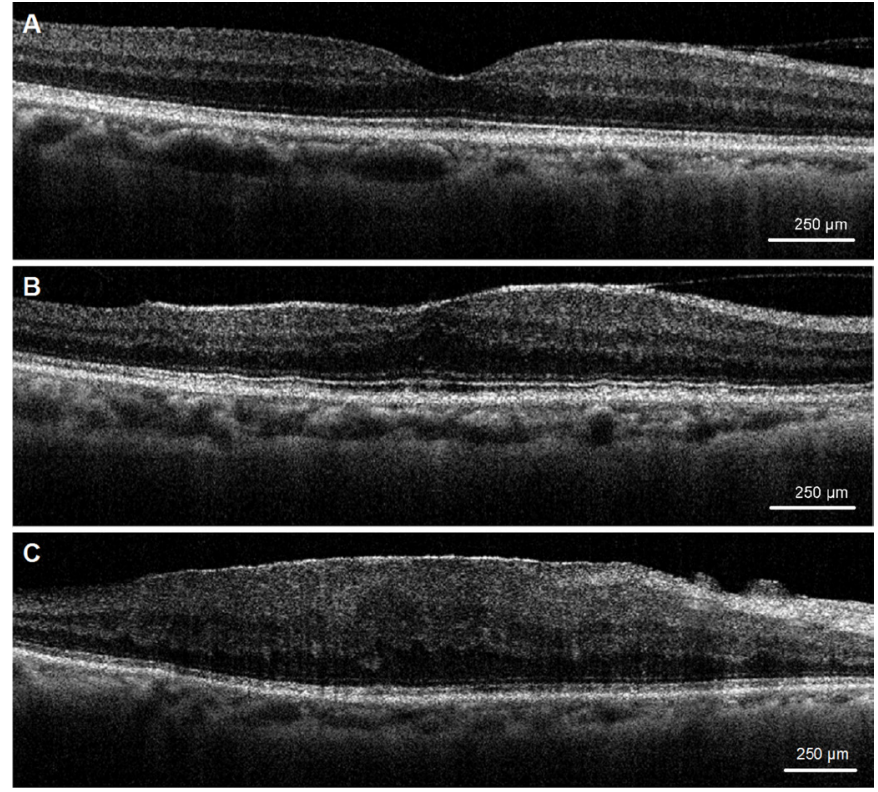

Figure 1 Optical coherence tomography photographs from the Liwan Eye Study showing examples of $(A)$ vitreomacular adhesion, $(\mathrm{B})$ vitreomacular traction and $(\mathrm{C})$ epiretinal membrane.

consent was obtained from all subjects after explaining the purpose of the study and the risks and benefits of the examination. From these subjects, those who underwent SD-OCT imaging between June 2013 and November 2013 were then included in the present analysis. This research adhered to the Declaration of Helsinki.

\section{Image acquisition}

SD-OCT imaging of the macula was performed by a trained technician using high-definition OCT (Model Ivue100, Optovue, Fremont, California, USA) with a retina map scanning protocol. Pupils were not dilated for OCT scannings. Images with a quality score less than 30 as self-evaluated by the OCT were retaken. If low quality of the image persisted despite multiple retakes, the image

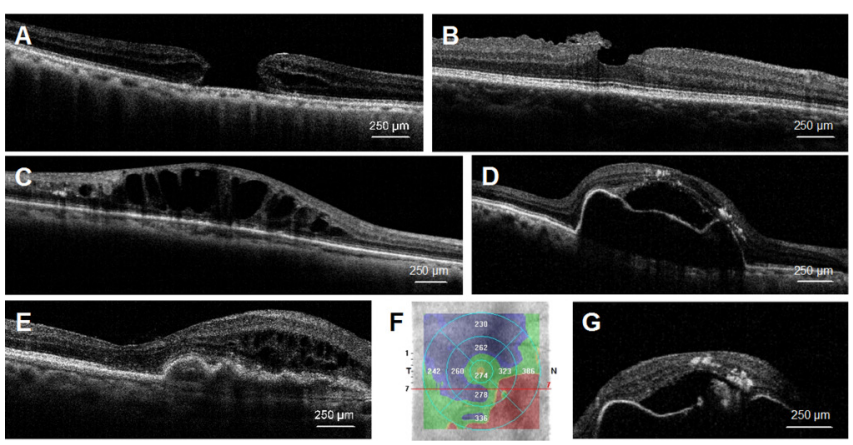

Figure 2 Optical coherence tomography photographs from the Liwan Eye Study showing examples of (A) full thickness macular hole, (B) lamellar macular hole, (C) macular retinoschisis, (D) retinal detachment, (E) macular oedema and (G) intraretinal hyper-reflective lesion. Figure part F depicts the macular tomographic map for the image displayed in figure part $E$ featuring regions with retinal thickness exceeding $300 \mu \mathrm{m}$.
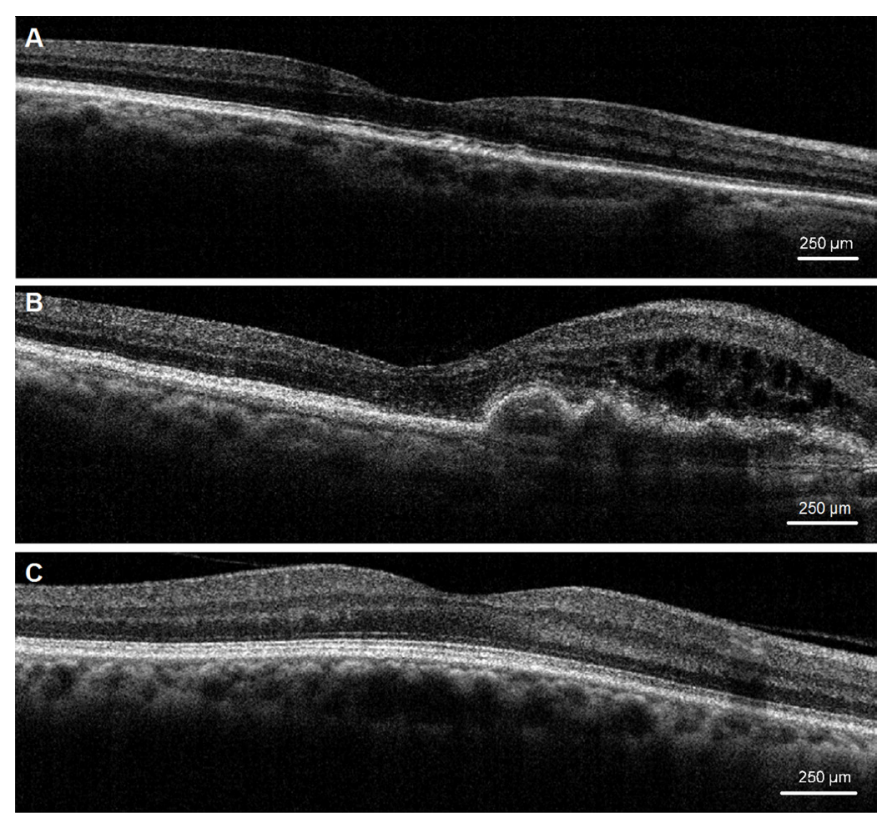

Figure 3 Optical coherence tomography photographs from the Liwan Eye Study showing examples of (A) retinal pigment epithelium thickening, (B) pigment epithelium detachment and $(\mathrm{C})$ dome-shaped macula.

was excluded from grading. For each graded eye, all scanning lines of the macular were analysed to assess for the presence of lesions. Images of the left eye were taken only if the examination of the right eye was not possible. Database entries were managed with EpiData V.3.0 or greater (EpiData Association, Odense, Denmark).

\section{Classification of lesions}

The layers of the macula in physiological conditions were identified on the basis of the classification system proposed by the International Nomenclature for Optical Coherence Tomography (IN•OCT) Panel, which that subdivides the retina into 18 layers progressing from the vitreoretinal interface towards the choroid-scleral interface ${ }^{10}$ For each OCT scan, the vitreoretinal interface, neurosensory retina (layers 1-13), RPE/Bruch's membrane complex (layer 14) and choroid (layers 15-18) were examined. A list of pathological lesions on SD-OCT proposed in this study are presented in table 1 . Additional grading details and standard photographs for each lesion are presented in online supplementary table 1 and figures 1-3.

\section{Grading of lesions}

Grading criteria and a set of standard photos for all OCT lesions assessed in this study are detailed in online supplementary materials. Briefly, lesions on each image were provided without other clinical information and were independently graded by trained observers into three mutually exclusive categories based on presence or absence of the lesion: 'None' (greater than 50\% certainty that a lesion is absent), 'Yes' (greater than $50 \%$ certainty that a lesion if present) or 'Cannot grade' (poor image quality or image obscured by mixture of lesions present). 
Table 2 Interobserver grading agreement on qualitative optical coherence tomography evaluations

\begin{tabular}{|c|c|c|c|c|c|}
\hline \multirow[b]{2}{*}{ Variable } & \multirow[b]{2}{*}{ Sample size } & \multirow[b]{2}{*}{ Graded (\%) } & \multicolumn{3}{|c|}{ Agreement } \\
\hline & & & Present & Absent & $\%$ Agreement \\
\hline \multicolumn{6}{|l|}{ Vitreoretinal interface } \\
\hline Vitreomacular adhesion & 679 & $673(99.1)$ & 66 & 571 & 94.7 \\
\hline Vitreomacular traction & 679 & $677(99.7)$ & 0 & 666 & 98.4 \\
\hline Epiretinal membrane & 679 & $677(99.7)$ & 66 & 548 & 90.7 \\
\hline \multicolumn{6}{|l|}{ Neurosensory retina } \\
\hline Full thickness macular holes & 679 & $678(99.9)$ & 1 & 676 & 99.7 \\
\hline Lamellar macular hole & 679 & $677(99.7)$ & 5 & 666 & 99.1 \\
\hline Macular retinoschisis & 679 & $676(99.6)$ & 7 & 651 & 97.3 \\
\hline Retinal detachment & 679 & $679(100)$ & 1 & 676 & 99.7 \\
\hline Macular oedema & 679 & $654(96.3)$ & 5 & 621 & 95.7 \\
\hline Intraretinal hyper-reflective lesion & 677 & $677(100)$ & 8 & 654 & 97.8 \\
\hline \multicolumn{6}{|l|}{ RPE/Bruch's complex } \\
\hline RPE thickening & 679 & $677(99.7)$ & 29 & 585 & 90.7 \\
\hline Pigment epithelium detachment & 678 & $678(100)$ & 3 & 662 & 98.1 \\
\hline Dome-shaped macula & 679 & $678(100)$ & 6 & 656 & 97.6 \\
\hline
\end{tabular}

RPE, retinal pigment epithelium.

Images from each subject were also graded on the ability to measure dome-shaped macula (DSM) height if such a lesion was present and choroidal thickness at three locations along the horizontal scan.

Two ophthalmologists served as observers to grade the OCT images. For qualitative OCT evaluations, interobserver agreement (percent of graded cases agreed between two assessments by different graders) was determined as a measure of inter-rater reliability. For quantitative measures of DSM height and choroidal thickness, descriptive statistics using the Student's t-test and intraclass correlation (ICC) scores were also calculated. ICC was determined with Stata V.12.0 using a two-way random effects model. For statistical analyses, a $\mathrm{P}$ value less than 0.05 was deemed significant.

\section{RESULTS}

A total of 679 OCT images from 679 subjects ( 379 women and 300 men; mean age $70.8 \pm 8.1$ years) were independently graded by two trained observers. On average, grading required approximately 5 min per image. Each lesion from table 1 was successfully scored as present or absent in over $96 \%$ of images with successful grading of all images for retinal detachments (RDs), intraretinal hyper-reflective lesions, pigment epithelium detachments (PEDs) and DSMs.

Table 2 presents the number of cases graded as present or absent by both observers for each lesion, as well as the interobserver agreement for each type of lesion assessed. For all 12 lesions in this study, per cent agreement between observers was over 90\%, ranging from $90.7 \%$ for ERMs and RPE thickenings to $99.7 \%$ for full thickness macular holes (FTMHs) and RDs.

Quantitative evaluations of DSM lesion height and choroidal thickness at three locations of the eye are shown in table 3. For all quantitative evaluations, the ICC score between the two graders was greater than 0.9 , indicating high reliability between graders for these measurements.

\section{DISCUSSION}

Based on recent definitions proposed for normal anatomic landmarks by the IN•OCT Panel, ${ }^{10}$ we defined and compared the scoring of 12 types of posterior eye lesions collected from a population of 679 subjects to evaluate the reliability of SD-OCT grading between clinicians. The highest per cent agreement scores were observed in the grading of FTMHs, lamellar macular holes and RDs, all of which are routinely diagnosed and monitored with the assistance of OCT in practice. ${ }^{12-14}$ Quantitative OCT evaluations also demonstrated a small but statistically significant difference in measurements between graders for DSM height and choroid thickness at nasal, temporal and subfoveal locations. The ability to accurately and non-invasively measure these parameters with OCT is important for both detecting new lesions and monitoring existing ones. In particular, SD-OCT may be of value to track conditions with associated choroidal thickness changes such as pachychoroid neovasculopathy and neovascular age-related macular degeneration. ${ }^{15} 16$ However, the potential for small discrepancies between independent graders measuring choroidal thickness with this modality should be noted. 
Table 3 Interobserver grading agreement on quantitative OCT evaluations

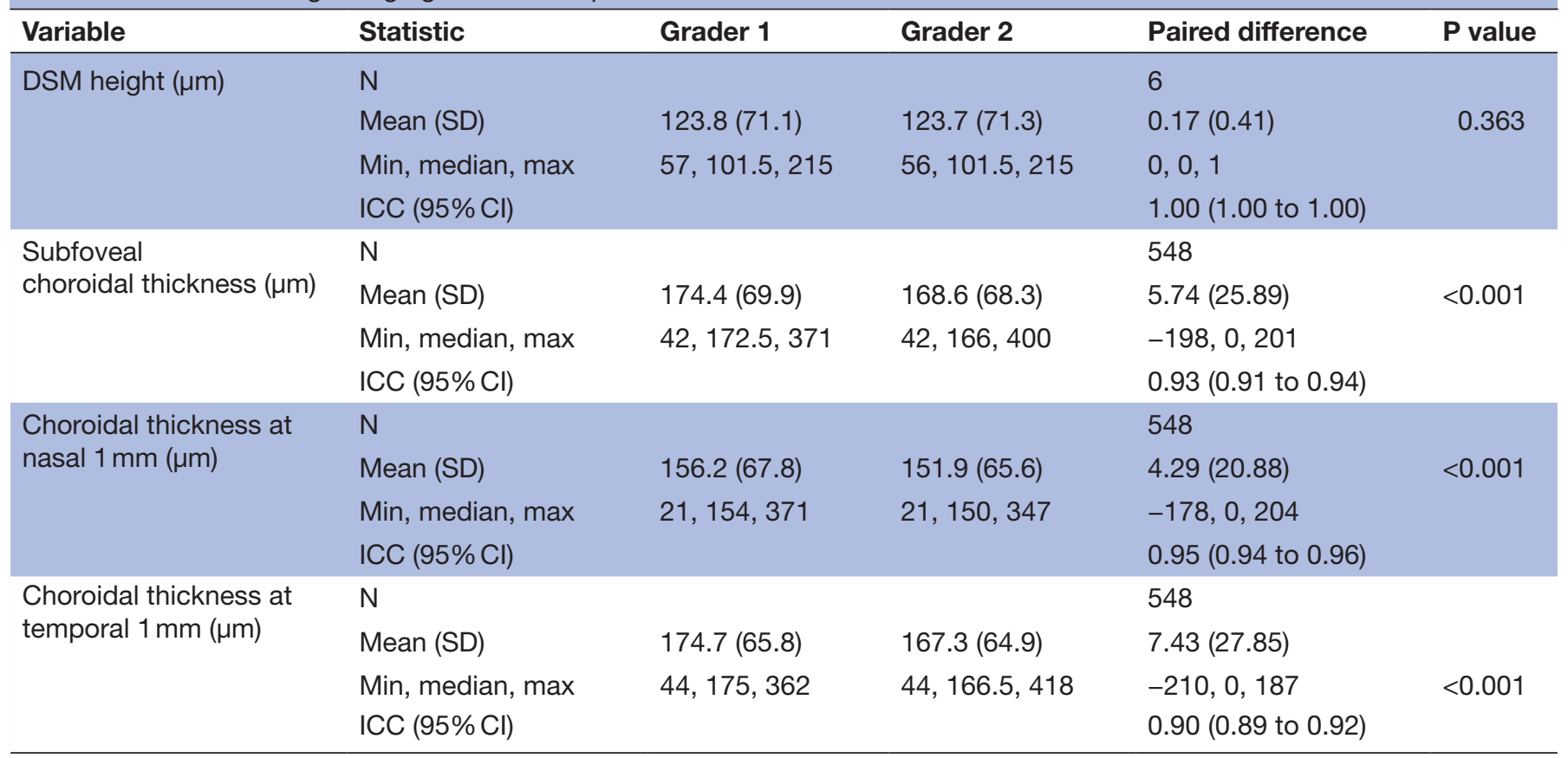

DSM, dome-shaped macula; ICC, intraclass correlation; OCT, optical coherence tomography.

From our analyses, the lowest per cent agreement scores were seen with assessments of macular oedema (MO), vitreomacular adhesions, ERMs and RPE thickening. The diversity of disease states leading to MO make it a challenging condition to diagnose, with prior attempts at characterising MO features by SD-OCT exhibiting good but imperfect agreement between independent image readers. ${ }^{9} 17$ Likewise, several forms of RPE thickening have been reported in the literature, yet the reliability among clinicians in using SD-OCT to identify this type of lesion has not previously been examined. For RPE thickening in particular, our grading criteria were based on examples from the literature of RPE proliferation, hypertrophy, pigmentation and migration changes, all of which notably lack a quantitative definition. $^{1819}$ The more subjective definition of RPE thickening on OCT may have made grading this lesion more difficult and may help explain its lower per cent agreement scores relative to other lesions in our study population. Further research should attempt to more precisely define criteria for these lesions to determine the reliability of these diagnoses from SD-OCT with greater confidence. Nonetheless, none of the lesions examined in this study showed per cent agreement between the graders of less than $90 \%$, underscoring the acceptable clinical utility of OCT for evaluating these changes in the eye.

Limitations of this study include the low prevalence in the enrolled population of FTMH, RD, PED and vitreomacular traction (VMT). VMT was not graded as present in any of the 679 subjects, dampening any conclusions that we can draw about this lesion. Limitations regarding the low prevalence of these lesions are to be expected as subjects were drawn from a population-based study. Given the growing adoption of SD-OCT in ophthalmic clinics and its increasing role in patient care, it is important that future studies verify the reliability of OCT image interpretation in daily clinical practice to avoid potential missed or erroneous diagnoses. Fortunately, in practice, the identification of these lesions is complemented by a range of imaging and diagnostic tools including fluorescein angiography, fundus autofluorescence and B-scan ultrasound. ${ }^{20}$ The collective findings from these tests would inform the results of SD-OCT, improving diagnostic accuracy and the possibility to intervene with appropriate treatment.

In summary, we proposed a standardised grading scheme for 12 different ocular pathological changes of the vitreoretinal interface, neurosensory retina and RPE/Bruch's complex by SD-OCT using recent IN•OCT consensus anatomic landmarks and assessed the agreement between trained clinicians in identifying these lesions. Our study demonstrates high concordance between graders in characterising SD-OCT images of these regions, providing validation for the continued use of SD-OCT as an ancillary test in the diagnosis of posterior eye pathology.

Contributors SKW, XG and MH designed the study, performed statistical analyses and drafted the manuscript. OX, YC, RL and WH collected the data used in the study. All authors read and approved the final manuscript.

Funding MH receives support from the National Natural Science Foundation of China (81125007) and the Fundamental Research Funds of the State Key Laboratory of Ophthalmology, Science and Technology Planning Project of Guangdong Province (92013B20400003). WH receives support from the National Natural Science Foundation of China (81570843H1204). MH receives support from the University of Melbourne at Research Accelerator Program and the 
CERA Foundation. The Centre for Eye Research Australia receives Operational Infrastructural Support from the Victorian State Government.

Competing interests None declared.

Patient consent Obtained.

Ethics approval This study was approved by the Zhongshan University Ethics Review Board and the Ethics Committee of Zhongshan Ophthalmic Center

Provenance and peer review Not commissioned; externally peer reviewed.

Data sharing statement Original data are available on request. Please contact the corresponding author for further information.

Open Access This is an Open Access article distributed in accordance with the Creative Commons Attribution Non Commercial (CC BY-NC 4.0) license, which permits others to distribute, remix, adapt, build upon this work non-commercially, and license their derivative works on different terms, provided the original work is properly cited and the use is non-commercial. See: http://creativecommons.org/ licenses/by-nc/4.0/

(c) Article author(s) (or their employer(s) unless otherwise stated in the text of the article) 2017. All rights reserved. No commercial use is permitted unless otherwise expressly granted.

\section{REFERENCES}

1. Huang D, Swanson EA, Lin CP, et al. Optical coherence tomography. Science 1991;254:1178-81.

2. Drexler W, Morgner U, Ghanta RK, et al. Ultrahigh-resolution ophthalmic optical coherence tomography. Nat Med 2001;7:502-7.

3. Chen TC, Cense B, Pierce MC, et al. Spectral domain optical coherence tomography: ultra-high speed, ultra-high resolution ophthalmic imaging. Arch Ophthalmol 2005;123:1715-20.

4. Zawadzki RJ, Jones SM, Olivier SS, et al. Adaptive-optics optical coherence tomography for high-resolution and high-speed 3D retinal in vivo imaging. Opt Express 2005;13:8532-46.

5. Ishikawa H, Stein DM, Wollstein G, et al. Macular segmentation with optical coherence tomography. Invest Ophthalmol Vis Sci 2005;46:2012-7.

6. Govetto A, Sarraf D, Figueroa MS, et al. Choroidal thickness in nonneovascular versus neovascular age-related macular degeneration: a fellow eye comparative study. Br J Ophthalmol 2017;101.

7. Kim KE, Oh S, Jeoung JW, et al. Spectral-domain optical coherence tomography in manifest glaucoma: its additive role in structural diagnosis. Am J Ophthalmol 2016;171:18-26.

8. Ruia S, Saxena S, Gemmy Cheung CM, et al. Spectral domain optical coherence tomography features and classification systems for diabetic macular edema: a review. Asia Pac J Ophthalmol 2016;5:360-7.

9. Heng LZ, Pefkianaki M, Pefianaki M, et al. Interobserver agreement in detecting spectral-domain optical coherence tomography features of diabetic macular edema. PLoS One 2015;10:e0126557.

10. Staurenghi G, Sadda S, Chakravarthy U, et al. Proposed lexicon for anatomic landmarks in normal posterior segment spectraldomain optical coherence tomography: the IN•OCT consensus. Ophthalmology 2014;121:1572-8.

11. He M, Foster PJ, Ge J, et al. Prevalence and clinical characteristics of glaucoma in adult Chinese: a population-based study in Liwan District, Guangzhou. Invest Ophthalmol Vis Sci 2006;47:2782-8.

12. Bottoni F, Carmassi L, Cigada M, et al. Diagnosis of macular pseudoholes and lamellar macular holes: is optical coherence tomography the "gold standard"? Br J Ophthalmol 2008;92:635-9.

13. Shimada N, Tanaka Y, Tokoro T, et al. Natural course of myopic traction maculopathy and factors associated with progression or resolution. Am J Ophthalmol 2013;156:948-57.
14. Panozzo G, Parolini B, Mercanti A. OCT in the monitoring of visual recovery after uneventful retinal detachment surgery. Semin Ophthalmol 2003;18:82-4.

15. Pang CE, Freund KB. Pachychoroid neovasculopathy. Retina 2015;35:1-9.

16. Shin JY, Kwon KY, Byeon SH. Association between choroidal thickness and the response to intravitreal ranibizumab injection in age-related macular degeneration. Acta Ophthalmol 2015;93:524-32.

17. Munk MR, Sacu S, Huf W, et al. Differential diagnosis of macular edema of different pathophysiologic origins by spectral domain optical coherence tomography. Retina 2014;34:2218-32.

18. Framme $C$, Walter $A$, Prahs $P$, et al. Structural changes of the retina after conventional laser photocoagulation and selective retina treatment (SRT) in spectral domain OCT. Curr Eye Res 2009;34:568-79.

19. Ho J, Witkin AJ, Liu J, et al. Documentation of intraretinal retinal pigment epithelium migration via high-speed ultrahigh-resolution optical coherence tomography. Ophthalmology 2011;118:687-93.

20. Keane PA, Sadda SR. Retinal imaging in the twenty-first century: state of the art and future directions. Ophthalmology 2014;121:2489-500.

21. Duker JS, Kaiser PK, Binder S, et al. The International Vitreomacular Traction Study Group classification of vitreomacular adhesion, traction, and macular hole. Ophthalmology 2013;120:2611-9.

22. Massin P, Allouch C, Haouchine B, et al. Optical coherence tomography of idiopathic macular epiretinal membranes before and after surgery. Am J Ophthalmol 2000;130:732-9.

23. Wilkins JR, Puliafito CA, Hee MR, et al. Characterization of epiretinal membranes using optical coherence tomography. Ophthalmology 1996;103:2142-51.

24. Flores-Moreno I, Arias-Barquet L, Vidal-Martí M, et al. The prevalence of vitreomacular interface pathology in a spanish tertiary hospital. Ophthalmologica 2016;235:179-83.

25. Bottoni F, Deiro AP, Giani A, et al. The natural history of lamellar macular holes: a spectral domain optical coherence tomography study. Graefes Arch Clin Exp Ophthalmol 2013;251:467-75.

26. Wu B, Deng J, Gao R, et al. Analysis of optic coherence tomography for congenital macular retinoschisis. Eye Sci 2011;26:80-4.

27. Sheth S, Dabir S, Natarajan S, et al. Spectral domain-optical coherence tomography study of retinas with a normal foveal contour and thickness after retinal detachment surgery. Retina 2010;30:724-32.

28. Wolfensberger TJ, Gonvers M. Optical coherence tomography in the evaluation of incomplete visual acuity recovery after maculaoff retinal detachments. Graefes Arch Clin Exp Ophthalmol 2002;240:85-9.

29. Wakitani $Y$, Sasoh M, Sugimoto M, et al. Macular thickness measurements in healthy subjects with different axial lengths using optical coherence tomography. Retina 2003;23:177-82.

30. Panozzo G, Parolini B, Gusson E, et al. Diabetic macular edema: an OCT-based classification. Semin Ophthalmol 2004;19:13-20.

31. Surguch V, Gamulescu MA, Gabel VP. Optical coherence tomography findings in idiopathic juxtafoveal retinal telangiectasis. Graefes Arch Clin Exp Ophthalmol 2007;245:783-8.

32. Roberts P, Baumann B, Lammer J, et al. Retinal pigment epithelial features in central serous chorioretinopathy identified by polarizationsensitive optical coherence tomography. Invest Ophthalmol Vis Sci 2016;57:1595.

33. Liang IC, Shimada N, Tanaka Y, et al. Comparison of clinical features in highly myopic eyes with and without a dome-shaped macula. Ophthalmology 2015;122:1591-600.

34. Ellabban AA, Tsujikawa A, Matsumoto A, et al. Three-dimensional tomographic features of dome-shaped macula by swept-source optical coherence tomography. Am J Ophthalmol 2013;155:320-8. 